\title{
Fuzzy goal programming to optimization the multi-objective problem
}

\author{
Azzabi Lotfi ${ }^{1, ~ *}$, Ayadi Dorra ${ }^{1}$, Bachar Kaddour ${ }^{2}$, Kobi Abdessamad ${ }^{1}$ \\ ${ }^{1}$ LASQUO/ISTIA/ University of Angers France \\ ${ }^{2}$ ESSCA Angers France
}

\section{Email address:}

lotfi_azzabi@yahoo.fr(L. Azzabi), dorra_ayadi@yahoo.fr (D. Ayadi), kaddour.bachar@essca.fr (K. Bachar), abdessamad.kobi@univ-angers.fr(A. Kobi),

\section{To cite this article:}

Azzabi. Lotfi, Ayadi Dorra, Bachar Kaddour, Abdessamad Kobi. Fuzzy Goal Programming to Optimization the Multi-Objective Problem. Science Journal of Applied Mathematics and Statistics. Vol. 2, No. 1, 2014, pp. 14-19. doi: 10.11648/j.sjams.20140201.12

\begin{abstract}
Many present-day problems are multi-objective in nature and their solution requires consideration of conflicting objectives. Usually, they have a number of potentially Pareto-optimal solutions. An extensive knowledge of the problem is required in discriminating between solutions, eliminating the unwanted ones and accepting the required solution(s) by a decision making process. It is well known that multi-objective optimization model had found a lot of important applications in decision making problems such as in economics theory, management science and engineering design. Because of these applications, a lot of literatures have been published to study optimality conditions, duality theories and topological properties of solutions of multi-objective optimization problems. In the case of optimization problems, the idea of regularizing a problem by adding a strongly convex term to the objective function can actually be treated back at least. The regularization technique proved to be an invaluable tool in the solution of ill-posed problems, and an enormous amount of work has been devoted to its study. In this paper, a Multi-objective Optimization Problems formulation based on a Goal Programming Methods solves the multi-objective problem which can tackle relatively large test systems. This method is based on optimization of the most preferred objective and considering the other objectives as constraints.
\end{abstract}

Keywords: Multicriteria Approach, Multi-Objective Optimization Problems, Fuzzy Goal Programming

\section{Introduction}

Optimization of a single objective oversimplifies the pertinent objective function in some potential mathematical programming application situations. These two statements introduce the general topic of multi-objective programming (1). Multi-objective programming involves recognition that the decision maker is responding to multiple objectives. Generally, objectives are conflicting, so not all objectives can simultaneously arrive at their optimal levels. Multi-objective programming formally permits formulations where:

a) Solutions are generated which are as consistent as possible with target levels of goals;

b) Solutions are identified which represent maximum utility across multiple objectives;

c) Solution sets are developed which contain all non-dominated solutions.

Multiple objectives can involve such considerations as leisure, decreasing marginal utility of income, risk avoidance, preferences for hired labor, and satisfaction of desirable, but not obligatory, constraints.

An assumed utility function is used to choose appropriate solutions. Several fundamentally different utility function forms have been used in multi-objective models.

\section{Multi-Objective Optimization}

\subsection{Principle}

Multi-objective optimization is a natural extension of the traditional optimization of a single-objective function. If the multi-objective functions are commensurate, or non-competing minimizing one-objective function minimizes all criteria and using traditional optimization techniques can solve the problem (2). However, if the objective functions are incommensurate, or competing, then minimization of one objective functions requires a 
compromise in another objective function. The competition between multi-objective functions gives rise to the distinguishing difference between multi-objective optimization and traditional single-objective optimization. This fact causes lack of complete order for multi-objective optimization problems (3).

It is clear from the above discussion that a multi-objective optimization problem usually has a set of Pareto-optimal solutions, instead of one single optimal solution 2. Thus, the objective in a multi-objective optimization is different from that in a single-objective optimization. In multi-objective optimization the goal is to find as many different Pareto-optimal (or near Pareto-optimal) solutions as possible. Since classical optimization methods work with a single solution in each iteration (4), in order to find multiple Pareto-optimal solutions they are required to be applied more than once, hopefully finding one distinct Pareto-optimal solution each time, (5); (6); (7); (8).

Before we discuss the problem features that may cause multi-objective difficulty, let us mention a couple of matters. First, we consider all objectives to be of minimization type. It is worth mentioning that identical properties as discussed here may also exist in problems with mixed optimization types (some are minimization and some are maximization). The concept of non-domination among solutions addresses only one type of problem.

\subsection{Formulation Multi-Objective Problem}

Consider a multi-objective optimization problem with $\mathrm{p}$ criteria or objectives where $X$ denotes the finite set of feasible solutions. Each solution $x \in X$ is represented in the criterion space by its corresponding criterion Vector $f(x)=\left(f_{1}(x), \ldots \ldots \ldots, f_{p}(x)\right)$. We assume in the following that each criterion has to be maximized.

From these $p$ criteria, the dominance relation defined on $X$, denoted by $\underline{\Delta}$, states that a feasible solution $x$ dominates a feasible solution $x^{\prime}, x \underline{\Delta} x^{\prime}$ if and only if $f_{i}(x) \geq f_{i}\left(x^{\prime}\right)$ for $i=1, \ldots, p$. We denote by $\Delta$ the asymmetric part of $\underline{\Delta}$. A solution $\mathrm{x}$ is efficient if and only if there is no other feasible solution $x^{\prime} \in X$ such that $x^{\prime} \Delta x$, and its corresponding criterion vector is said to be non-dominated. Thus, the efficient set is defined as $E(x)=\left\{x \in X: \forall x^{\prime} \in X,\left(x^{\prime} \Delta x\right)\right\}$ (9).

$$
E(x)=\left\{x \in X: \forall x^{\prime} \in X,\left(x^{\prime} \Delta x\right)\right\} \quad \text { The set of }
$$

non-dominated criterion vectors, which corresponds to the image of the efficient set in the criterion space, is denoted by $N D$. Since the efficient set can contain different solutions corresponding to the same criterion vector, any subset of $E(x)$ that contains one and only one solution for every non-dominated criterion vector is called a reduced efficient set. Observe that $X^{\prime} \subseteq X$ is a reduced efficient set if and only if it is a covering and independent set of $X$ with respect to $\underline{\Delta}$. We recall that, given $\succeq$ a binary relation defined on a finite set $A$,

- $B \subseteq A$ is a covering (or dominating) set of $A$ with respect to $\succ$ if and only if for all $a \in\{A, B\}$, there exists $b \in B$ such that $b \succeq a$.

- $B \subseteq A$ is an independent (or stable) set with respect to $\succeq$ if and only if for all $b, b^{\prime} \in B, b \neq b^{\prime}$, not $\left(b \succeq b^{\prime}\right)$.(10)

\section{Basic Structure of Fuzzy Goal Programming}

\subsection{Principle}

Goal programming (GP) Models were originally introduced by Charnes and Cooper in early 1961 for a linear model. This approach allows the simultaneous solution of a system of complex objectives and the solution of the problem requires the establishment among multiple objectives.

The principal concept for linear GP is to the original turn multiple objectives into specific numeric goal for each objective. The objective function is then formulated and a solution is sought which minimizes the weighted sum of deviations from their respective goal. In general, GP models consist of three components: an objective function, a set of goal constraints, and non-negativity requirements. However, the target value associated with each goal could be fuzzy in the real-world application. The fuzzy sets theory is recurrently used in recent research. A fuzzy set $A$ can be characterized by a membership function, usually denoted $\mu$, which assigns to each object of a domain its grade of membership in $A$ (11). The more an element or object can be said to belong to a fuzzy set $A$, the closer to 1 is its grade of membership. Various types of membership functions can be used to support the fuzzy analytical framework although the fuzzy description is hypothetical and membership values are subjective (12).

Membership functions, such as linear, piecewise linear, exponential, and hyperbolic functions, were used in different analysis. In general, the non-increasing and non-decreasing linear membership functions are frequently applied for the inequalities with less than or equal to and greater than or equal to relationships, respectively. Since the solution procedure of the fuzzy mathematical programming is to satisfy the fuzzy objective, a decision in a fuzzy environment is thus defined as the intersection of those membership functions corresponding to fuzzy objectives (13). Hence, the optimal decision could be any alternative in such a decision space that can maximize the minimum, represented by those corresponding membership functions (14).

The integrated use at GP and fuzzy sets theory has already been reported in the literature, (15), (16), (17), (18), (19), (20), (21), (22), (23) further integrated several fuzzy linear and multi-objective programming techniques. 


\subsection{Goal Programming Problem}

The goal programming is a multi-objective mathematical programming model that attempts to produce an optimum solution for several conflicting objectives. The optimal solution would be the most satisfactory solution within a set of feasible solutions (24). The GP model is a more flexible technique that is relatively easy to understand and utilize. There has been previous applications of GP in industrial and production settings however none of the early uses of GP explicitly incorporates the decision maker's preferences.

While we apply GP to capacity assessment problem we will consider preferences of the decision maker explicitly expressed through the concept of satisfaction function (25) and (26).

The overall purpose of GP is to minimize the deviations between the achievement of goals and their aspiration levels. A typical GP is expressed as follows:

Minimize

$$
\sum_{i=1}^{k}\left|F_{i}(x)-g_{i}\right|
$$

Subject to

$$
x \in X=\left\{x \in R^{n} ; A_{X} \leq b ; x \geq 0\right\}
$$

Where $F_{i}$ the linear is function of the $i$ th goal and $g_{i}$ is the aspiration level of $i$ th goal. Let

$$
F_{i}(x)-g_{i}=d_{i}^{+}-d_{i}^{-} ; d_{i}^{+}, d_{i}^{-} \geq 0
$$

Equation (1) can be formulated as follows:

Minimize

$$
\sum_{i=1}^{k}\left|d_{i}^{+}+d_{i}^{-}\right|
$$

Subject to

$$
\begin{gathered}
F_{i}(x)-d_{i}^{+}+d_{i}^{-}-g_{i}=0 ; i=1,2, \ldots \ldots . k \\
x \in X=\left\{x \in R^{n} ; A_{X} \leq b ; x \geq 0\right\}
\end{gathered}
$$

Where $d_{i}^{+} \geq 0 ; d_{i}^{-} \geq 0$ are, respectively under deviations of $i$ th goal.

\subsection{Formulation Fuzzy Goal Programming}

Narasimhan, 1980 were the first to give a Fuzzy Goal Programming (FGP) formulation by using the concept of the membership functions. These functions are defined on the interval $[0,1]$. So, the membership function for the i-th goal has a value of 1 when this goal is attained and the decision multicriteria is totally satisfied; otherwise the membership function assumes a value between 0 and 1 . Linear membership functions are used in literature and practice more than other types of membership functions. For the above three types of fuzzy goals linear membership functions are defined and depicted as follows (Fig. 1):

Where $L_{k}\left(\right.$ or $\left.u_{k}\right)$ is lower tolerance limit for $k$.th fuzzy goal $G_{k}(x)$ They are either subjectively chosen by decision makers or tolerances in a technical process.

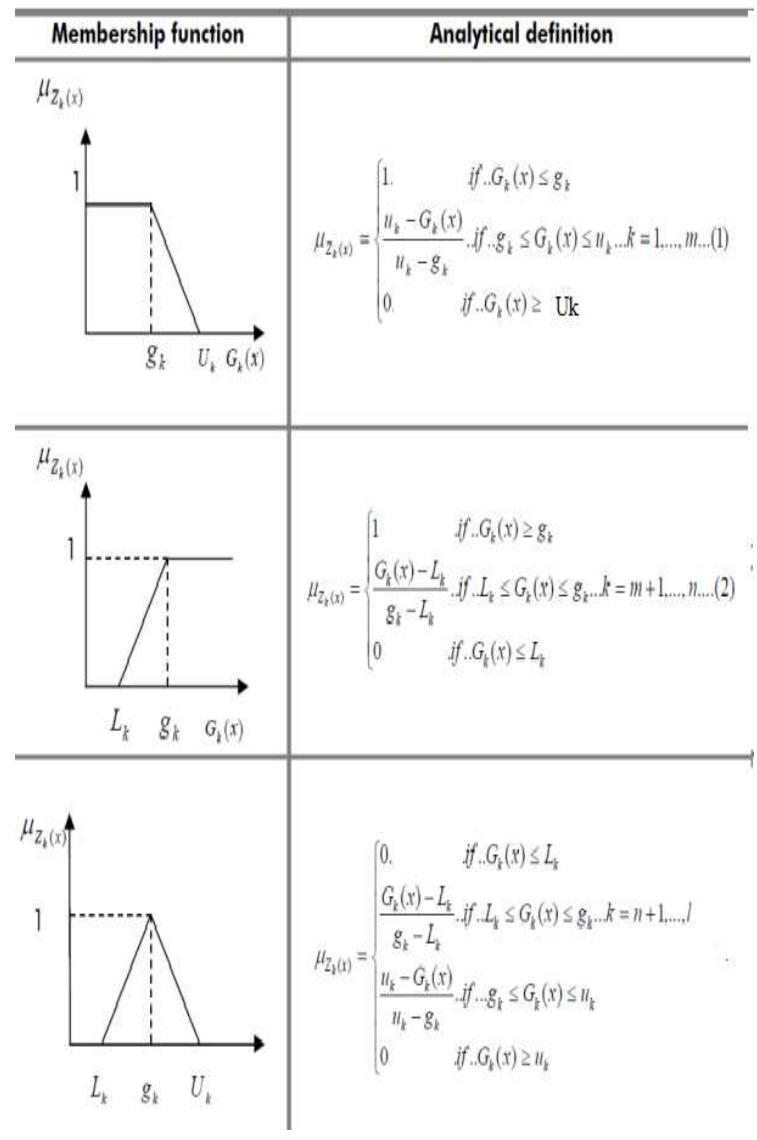

Figure 1. Fuzzy Goals Linear Membership Functions

\subsection{Fuzzy Linguistic for Determining the Degree of Achievement}

The determination of a desirable achievement degree for a goal could be a difficult task for a decision making in a fuzzy environment when using method by (22). For assessing desirable achievement degrees imprecisely, a useful method is to use linguistic terms such as "Low Important", "Somewhat High Important", and "Very High Important" and so on to verbally describe the importance of each fuzzy goal. The associated membership functions are then defined. We can define $u_{I}(\alpha)$ to represent the membership function of each linguistic values about the importance of different objectives, where $u_{I}(\alpha) \in[0,1]$, and $\alpha$ denotes the variable taking an achievement degree in the interval of $\left[\alpha_{\text {min }}, \alpha_{\text {max }}\right], 0 \leq \alpha_{\text {min }} \leq \alpha_{\max } \leq 1$.

Then fuzzy numbers ranking methods can be used to map a membership function representing a fuzzy goal's importance to a real number in the range of $[0,1]$. The real number obtained can be considered as the desirable 
achievement degree for the fuzzy goal.

We define $\mathrm{I}=$ \{Very Low Important $=$ VLI, Low Important $=$ LI, Somewhat Low Important $=$ SLI, Medium $=\mathrm{M}$, Somewhat High Important $=$ SHI, High Important $=$ HI, Very High Important $=\mathrm{VHI}\}$, as a set of linguistic values about the importance of different goals (Figure.2). shows the $u_{i}(\alpha)$ for this linguistic values. Triangular fuzzy numbers corresponding to these linguistic values are: $\mathrm{VLI}=(0,0,10 \%), \mathrm{LI}=(5 \%, 15 \%, 25 \%), \mathrm{SLI}=(20 \%, 32.5 \%$, $45 \%), \mathrm{M}=(40 \%, 50 \%, 60 \%), \mathrm{SHI}=(55 \%, 67.5 \%, 80 \%), \mathrm{HI}$ $=(75 \%, 85 \%, 95 \%), \mathrm{VHI}=(90 \%, 100 \%, 100 \%)$.

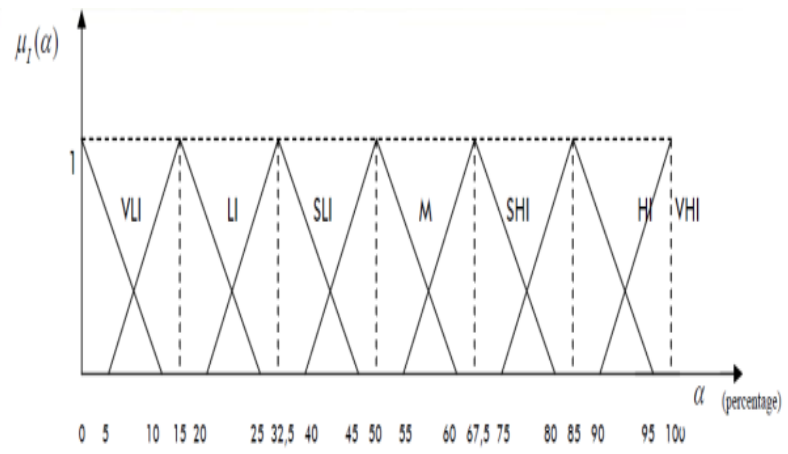

Figure 2. Functions for linguistic values about the importance of different objectives

\section{Numerical Example with Fuzzy Goal Programming}

\subsection{Data Description}

In this section, we discuss the case of a joint shipping company, which seeks to maximize the profitability of its customer services and this by minimizing the number of road accidents for each vehicle (bus). A bus incurs three major networks: 1: city university, 2: town center and 3: industrial zone.

As the following Parameters and constants:

dit : Travel time of driver $i$ for period $j$;

cit: Assure cost of the accident the driver i in period $\mathrm{j}$;

rt: Regular time work force cost per employee hour in period t;

vit : Speed incurred by the driver $\mathrm{i}$ during period $\mathrm{j}$;

Kit: Level of knowledge of road network driver $i$ in period t;

Iio: number of accidents made by the drivers $\mathrm{i}$ in period $\mathrm{t}$ -1 ;

T: planning horizon paths of travel;

$\mathrm{N}$ : Total number of drivers;

Pit: number of conductor $i$ in period $t$;

Iit: number of accidents made by the drivers $i$ in period $t$;

Ht: worker hired in period $t$;

Ft: workers laid off in period t;
Iit. Min: minimum conductor $\mathrm{i}$ in period $\mathrm{t}$;

$\mathrm{Wt}$ : Total number of buses during the period $\mathrm{t}$;

WMin: Minimum number of buses available in period t;

WMax: Maximum number of buses available in period $t$;

The proposed model implementation in the company has the following conditions:

1. There is a six period planning horizon,

2. Three road networks,

3. Number minimum of accidents made by the drivers $i$ in period $\mathrm{t}-1 \mathrm{I}: \mathrm{I} 10=18$ accidents in the network $1, \mathrm{I} 20$ $=10$ accidents in the network $2, \mathrm{I} 30=33$ accidents in the network 3.

4. Minimum number of accident during the period $t$ of drivers $i$ is 30 non-fatal accident.

5. Cost of repair or re-purchase bus accident per bus are respectively $10386 £$ and $27900 £$.

6. The linguistic values about the importance of objectives are: Very igh Important = VHI, High Important $=\mathrm{HI}$, Medium $=\mathrm{M}$. respectively. And assumed that we have moderate decision maker, with $\alpha=0.5$.

7. The cost of one driver in the bus of three network during the $\mathrm{t}$ period is $\mathrm{Rt}=2500 £ / \mathrm{man}$.

8. Minimum number of buses available in period $t$ is WMin $=50$ bus of three network.

9. Minimum number of buses available in period $t$ is WMax $=80$ bus of three network.

10. The initial number of bus WMin $=130$ bus of three network.

11. Number maximum of accidents made by the drivers $i$ in period $\mathrm{t}$ of three networks is 80 accidents.

Table 1. The basic data provided in the three networks

\begin{tabular}{|c|c|c|c|c|c|}
\hline & Period & dit & vit & cit & Kit \\
\hline \multirow{6}{*}{ Network 1} & 1 & 210 & $50 \mathrm{~km} / \mathrm{h}$ & $2500 £$ & 21 \\
\hline & 2 & 120 & $40 \mathrm{~km} / \mathrm{h}$ & $2300 £$ & 23 \\
\hline & 3 & 150 & $30 \mathrm{~km} / \mathrm{h}$ & $2450 £$ & 24 \\
\hline & 4 & 120 & $50 \mathrm{~km} / \mathrm{h}$ & $1987 £$ & 26 \\
\hline & 5 & 72 & $80 \mathrm{~km} / \mathrm{h}$ & $2365 £$ & 23 \\
\hline & 6 & 138 & $40 \mathrm{~km} / \mathrm{h}$ & $2789 £$ & 24 \\
\hline \multirow{6}{*}{ Network 2} & 1 & 150 & $50 \mathrm{~km} / \mathrm{h}$ & $2500 £$ & 21 \\
\hline & 2 & 120 & $50 \mathrm{~km} / \mathrm{h}$ & $1900 £$ & 25 \\
\hline & 3 & 138 & $50 \mathrm{~km} / \mathrm{h}$ & $2450 £$ & 22 \\
\hline & 4 & 210 & $60 \mathrm{~km} / \mathrm{h}$ & $1987 £$ & 21 \\
\hline & 5 & 72 & $40 \mathrm{~km} / \mathrm{h}$ & $2365 £$ & 24 \\
\hline & 6 & 150 & $50 \mathrm{~km} / \mathrm{h}$ & $1789 £$ & 25 \\
\hline \multirow{6}{*}{ Network 3} & 1 & 120 & $50 \mathrm{~km} / \mathrm{h}$ & $2500 £$ & 22 \\
\hline & 2 & 150 & $70 \mathrm{~km} / \mathrm{h}$ & $2380 £$ & 24 \\
\hline & 3 & 138 & $50 \mathrm{~km} / \mathrm{h}$ & $2450 £$ & 25 \\
\hline & 4 & 210 & $50 \mathrm{~km} / \mathrm{h}$ & $1987 £$ & 26 \\
\hline & 5 & 120 & $50 \mathrm{~km} / \mathrm{h}$ & $1865 £$ & 25 \\
\hline & 6 & 72 & $80 \mathrm{~km} / \mathrm{h}$ & $2789 £$ & 21 \\
\hline
\end{tabular}




\subsection{Formulate and Solving Problem}

Construct the membership functions:

The linear membership function of each objective function is determined by asking the decision making to specify the interval $\left[g_{k} \ldots u_{k}\right]$ of the objective values, and also to specify the equivalence of these objective values as a membership value in the interval $[0,1]$. The linear and continuous membership function is found to be suitable for quantifying the fuzzy spiration levels.

Function 1: Minimize the total assure cost of the accident

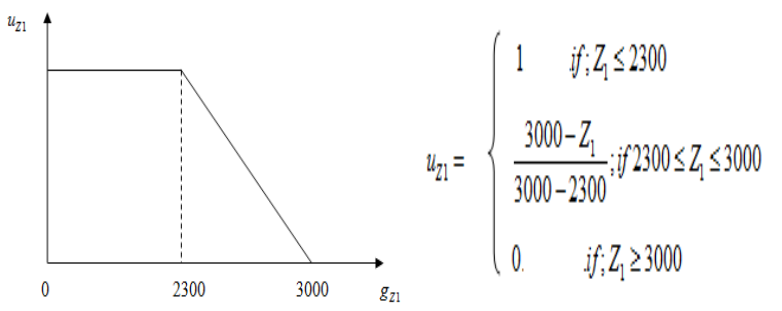

Figure 3. Membership function $Z_{1}$

Function 2: Minimize the total speed

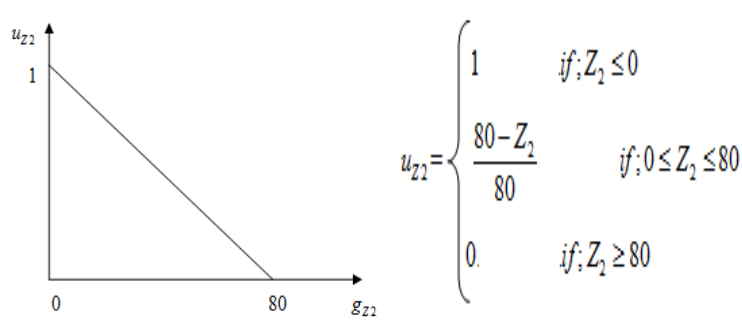

Figure 4. Membership function $Z_{2}$

Function 3: minimization of changes in working time

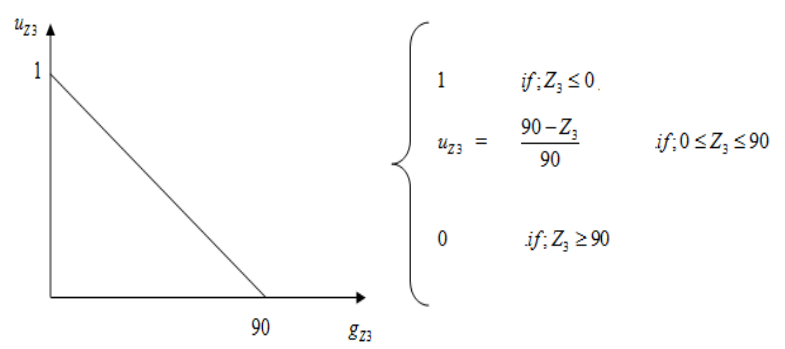

Figure 5. Membership function $Z_{3}$

Then, the objective functions are:

Max

$$
f(u)=\sum_{k=1}^{3} u_{k}
$$

Subject to :

$$
\begin{aligned}
& u_{1} \leq \frac{3000-Z_{1}}{7000} \\
& u_{2} \leq \frac{80-Z_{2}}{80} \\
& u_{3} \leq \frac{90-Z_{3}}{90}
\end{aligned}
$$

$$
\begin{gathered}
P_{i t}-K_{i t} \times W_{t} \leq 0 \\
P_{i t}+I_{i 0}-I_{i t}=d_{i t} \\
W_{t}-W_{0}-H_{t}+F_{t} \leq W_{t} \\
W_{\min } \leq W_{t} \leq W_{\max } \\
\sum_{i=1}^{3} I_{i t} \leq 61 \\
I_{i t} \geq 30 \\
I_{10}=18 ; \quad I_{20}=10 ; I_{30}=33 \\
u_{1} \geq 0.72 \\
u_{2} \geq 0.85 \\
u_{3} \geq 0.50 \\
I_{i t}, u_{1}, u_{2}, u_{3} \geq 0 \\
i=1,2,3 \\
t=1,2,3,4,5,6
\end{gathered}
$$

\begin{tabular}{|c|c|c|c|c|}
\hline Period & Networks & $I_{i t}$ & $W_{i t}$ & $P_{i t}$ \\
\hline \multirow{3}{*}{0} & 1 & 18 & \multirow{3}{*}{80} & \multirow[t]{3}{*}{-} \\
\hline & 2 & 10 & & \\
\hline & 3 & 33 & & \\
\hline \multirow{3}{*}{1} & 1 & 0 & \multirow{3}{*}{80} & \multirow{3}{*}{55} \\
\hline & 2 & 0 & & \\
\hline & 3 & 30 & & \\
\hline \multirow{3}{*}{2} & 1 & 18 & \multirow{3}{*}{80} & \multirow{3}{*}{55} \\
\hline & 2 & 0 & & \\
\hline & 3 & 10 & & \\
\hline \multirow{3}{*}{3} & 1 & 0 & \multirow{3}{*}{80} & \multirow{3}{*}{55} \\
\hline & 2 & 0 & & \\
\hline & 3 & 30 & & \\
\hline \multirow{3}{*}{4} & 1 & 0 & \multirow{3}{*}{80} & \multirow{3}{*}{55} \\
\hline & 2 & 10 & & \\
\hline & 3 & 18 & & \\
\hline \multirow{3}{*}{5} & 1 & 0 & \multirow{3}{*}{80} & \multirow{3}{*}{55} \\
\hline & 2 & 0 & & \\
\hline & 3 & 33 & & \\
\hline \multirow{3}{*}{6} & 1 & 0 & \multirow{3}{*}{80} & \multirow{3}{*}{55} \\
\hline & 2 & 0 & & \\
\hline & 3 & 30 & & \\
\hline
\end{tabular}

Solve problem:

The LINGO computer software package was used to run the programming model:

Table 2. Optimal solution model

Using Fuzzy Goal Programming to simultaneously minimize total road accident costs $\left(Z_{1}\right)$, carrying costs $\left(Z_{2}\right)$, and changes in Work force levels $\left(Z_{3}\right)$, yields total road accident cost of $2999 £$, and resulting achievement degrees for the three fuzzy goal $\left(u_{1}, u_{2}\right.$ and $\left.u_{3}\right)$ are 0,0 and 1 respectively, all of which satisfy the requirements of decision makers.

\section{Conclusion}

In this paper, we presented a road accident constraint in which data of three networks are fuzzy values and the objective function assumes multiple objectives. Then, non-linear constraints are linearized by defining and adding 
auxiliary constraints. Finally, the optimal solution of the problem is founded by solving the linear programming problem with fuzzy and crisp constraints and applying fuzzy programming technique.

Various fuzzy approaches have been proposed for the solution of multi-objective linear plus linear fractional programs problem and most of the approaches have min trual computational burden. Our approach is to give simple procedure for the solution of multi-objective fuzzy goal programming problems.

\section{References}

[1] B F. Blake, BA. McCarl., "Goal Programming via Multidimensional Scaling Applied to Sengalese Subsistence Farming: A Reply," American Journal of Agricultural Economics. 65 (1983):832-33.

[2] M. Baltes, R. Schneider, C. Sturm, and M. Reuss., "Optimal experimental design for parameter estimation in unstructured growth models," Biotechnology Progress, 10, $480\} 488(1994)$.

[3] LT. Biegler, J J. Damiano. GE. Blau., "Nonlinear parameter estimation: A case study comparison," A.I.Ch.E. Journal, 32, 29\}45(1986).

[4] K. Deb., " Optimization for engineering design: Algorithms and examples." Prentice-Hall, New Delhi, India (1995).

[5] C M. Fonseca, P J. Fleming., " An overview of evolutionary algorithms in multiobjective optimization," Evolutionary Computation, 3:1-16 (1995).

[6] J. Horn, N. Nafploitis, and D E. Goldberg., " A niched Pareto genetic algorithm for multiobjective optimization, " In Michalewicz, Z., editor, Proceedings of the First IEEE Conference on Evolutionary Computation, pages 82-87, IEEE Service Center, Piscataway, New Jersey, (1994).

[7] N. Srinivas, K. Deb., "Multi-Objective function optimization using non-dominated sorting genetic algorithms," Evolutionary Computation, 2(3):221-248, (1995).

[8] E. Zitzler, L. Thiele., "Multiobjective optimization using evolutionary algorithms - A comparative case study,. " In Eiben, A. E., B"ack, T., Schoenauer, M. and Schwefel, H.-P., editors, Parallel Problem Solving from Nature, V, pages 292-301, Springer, Berlin, Germany, (1998).

[9] J. Teng, G. Tzeng., "A multiobjective programming approach for selecting non-independent transportation investment alternatives," Transportation Research-B, 30(4):201-307, 1996, (1996).

[10] M. Ehrgott., "Multicriteria optimization,” LNEMS 491. Springer, Berlin, 2005.

[11] L A. Zadeh,. " Fuzzy Sets, Information and Control, " 8, 1965, pp. 338-353.
[12] M. Belmokaddem, M. Mekidiche, A. Sahed.," Application of a fuzzy GOAL PROGRAMMING approach with different importance and priorities to aggregate production planning," Journal of Applied Quantitative Methods, (2009).

[13] HJ. Zimmermen., "Fuzzy programming and linear programming with several objective functions, " Fuzzy Sets and Systems, 1, 1978, pp. 45-56, 1978.

[14] HJ. Zimmermann., "Applications of fuzzy sets theory to mathematical programming," Information Science, 35, 1985, pp. 29-58, (1985).

[15] E.L, Hannan., "Linear programming with multiple fuzzy goals," Fuzzy Sets and Systems, 6,1981-a, pp. 235-248, (1981).

[16] E.L, Hannan., "On Fuzzy Goal Programming, " Decision Sciences 12, 1981-b, pp. 522-531, (1981).

[17] H. Leberling., "On finding compromise solutions in multi criteria problems using the fuzzy min-operator, " Fuzzy Sets and Systems, 6, 1981, pp. 105-118, (1981).

[18] MK. Luhandjula., "Compensatory operations in fuzzy programming with multiple objectives, " Fuzzy Sets and Systems, 8, 1982, pp. 245-252, (1982).

[19] PA. Rubin, R. Narasimhan., "Fuzzy goal programming with nested priorities," Fuzzy Sets and Systems, 14, 1984, pp. $115-129,(1984)$

[20] R N. Tiwari, S. Dharmar, and J R. Rao., "Fuzzy goal programming - An additive model," Fuzzy Sets and Systems, 24, 1987, pp. 27-34, (1987).

[21] H F. Wang, C. C, Fu., "A generalization of fuzzy goal programming with preemptive structure,"Computers and Operations Research, 24, 1997, pp. 819-828, (1997).

[22] L H. Chen, F C. Tsai., "Fuzzy goal programming with different importance and priorities, " European Journal of Operational Research, 133, 2001, pp. 548-556, (2001).

[23] M A. Yaghoobi, M. Tamiz., " A method for solving fuzzy goal programming problems based on MINMAX approach,"European Journal of Operational Research, 177, pp. 1580-1590, (2007).

[24] B. Aouni, O. Kettani., "Goal Programming Model: A Glorious History and a Promising Future", European Journal of Operational Research, Vol. 133, No. 2, 1-7, (2001).

[25] J-M. Martel, B. Aouni., "Incorporating the Decision-maker's Preferences in the Goal Programming Model", Journal of Operational Research Society, Vol.41, 1121-1132, (1990).

[26] J M. Martel, B. Aouni., "Incorporating the Decision-maker's Preferences in the Goal Programming Model with Fuzzy Goals Values: A new Formulation", Lecture Notes in Economics and Mathematical Systems, Springer-Verlag, (1996). 